Homology, Homotopy and Applications, vol.8(2), 2006, pp.91-103

\title{
HOMOLOGY WITH LOCAL COEFFICIENTS AND CHARACTERISTIC CLASSES
}

\author{
ROBERT GREENBLATT
}

(communicated by Donald M. Davis)

\begin{abstract}
In this thesis, we are concerned with the study of cohomology with local coefficients and applications to (non-orientable) real vector bundles. The thesis consists of an introduction and three separate sections. The introduction gives some motivation for considering cohomology with local coefficients and an outline of the results obtained. The first section deals with a general discussion of such cohomology groups and contains a Künneth Theorem for such groups. The second section is devoted to some computations which are needed later and the final section gives a complete description of the integral cohomology of the spaces $B O(n)$ and $B S O(n)$.
\end{abstract}

\section{Introduction}

A great deal of information has been obtained about real vector bundles by studying various "characteristic classes". (See, for example, $[\mathbf{2}]$ or $[\mathbf{9}]$.) Usually, these results are about the mod 2 classes of an arbitrary bundle or the integral classes of an orientable bundle. The study of the integral classes of a non-orientable bundle $[\mathbf{1 2}]$ is complicated by the fact that these are "twisted" cohomology classes. (See Section 3 for a definition).

That the situation is more difficult in the case of non-orientable bundles is illustrated by the following example. It is known $[\mathbf{9}]$, [14], that the normal bundle to an imbedding of an orientable manifold in a Euclidean space always has a trivial Euler class. However, an example due to Whitney [15] shows that this is not true for non-orientable manifolds.

It was our original purpose to study non-orientable real vector bundles by studying the twisted integral characteristic classes. We do this by the familiar technique of studying the universal classes. Explicitly, if $B O(n)$ is the classifying space of the real orthogonal group $O(n)$ and $\mathfrak{Z}$ is the local system of twisted integer coefficients on $B O(n)$ (see [11] or [14]), we describe completely the groups $H^{q}(B O(n), \mathfrak{Z})$ as well as $H^{*}(B O(n), Z)$. This is done in Section 3. The first two sections are devoted to a study of properties of cohomology with local coefficients which are needed in

Based on the author's Ph.D. thesis, Yale, 1963.

Received February 24, 2006, revised April 16, 2006; published on July 21, 2006.

2000 Mathematics Subject Classification: 55R25, 55R40.

Key words and phrases: local cohomology, characteristic classes.

Copyright (C) 2006, International Press. Permission to copy for private use granted. 
Section 3. In particular, Section 1 is concerned with proving a Künneth formula for cohomology with coefficients in an arbitrary local system.

Section 2 is concerned with the special case of local coefficients with fiber $Z$, the group of integers. Here, we give a characterization of the "twisted" Bockstein homomorphisms induced by the exact coefficient sequence $0 \rightarrow Z \rightarrow Z \rightarrow Z_{2} \rightarrow 0$ and generalize a result of Massey [8] on the Gysin sequence of a vector bundle.

\section{Acknowledgements}

I take this opportunity to express my gratitude to Professor W.S. Massey who aided me immeasurably in the preparation of this thesis. It was upon his suggestion that I began working on this problem, and his encouragement and insight were a constant inspiration.

\section{Homology with local coefficients}

The main result of this section is a Künneth formula for homology and cohomology of spaces with coefficients in a local system of groups. For a definition of such homology groups, the reader is referred to Steenrod's paper [11]. Equivalently, a local system of coefficients is a locally constant sheaf [4, exposé 14] or, if the base space is "nice", it is a bundle of coefficients [12]. The remainder of the section is devoted to the special case of "twisted integer" cohomology and some results are developed which are needed in subsequent parts of the paper.

\subsection{The Künneth Theorem}

Let $K$ be an arcwise connected space and $G$ an abelian group. We recall that a local system of coefficients [11] with fiber $G$ over $K$ is an element

$$
v \in \operatorname{Hom}\left(\pi_{1}(K), \Gamma(G)\right),
$$

where $\pi_{1}(K)$ is the fundamental group of $K$ and $\Gamma(G)$ is the group of automorphisms of $G$. We shall write $G_{v}$ for such a local system and consider $G$ as a right $\pi=\pi_{1}(K)$ module with the action induced by $v$.

Theorem 1.1 (Eilenberg). If $K$ is an arcwise connected space with universal covering space $\tilde{K}$, then there exist natural isomorphisms

$$
H^{q}\left(K, G_{v}\right) \approx H_{q}\left(G \otimes_{\pi} C(\tilde{K})\right)
$$

and

$$
H_{q}\left(K, G_{v}\right) \approx H^{q}\left(\operatorname{Hom}_{\pi}(C(\tilde{K}), G)\right),
$$

where tensor products are taken over the group ring $Z(\pi)$.

Proof. The groups on the right are the "equivariant" homology groups of $\tilde{K}$ with $\pi$ operating on the left as covering transformations. For details, see [6, Theorem 24.1] or $[\mathbf{3}$, p. 355]. 
Theorem 1.2. Let $X$ and $Y$ be positive chain complexes with boundaries of degree -1 . If either $X$ or $Y$ is free abelian, then there exists an exact sequence

$$
0 \rightarrow H(X) \otimes H(Y) \rightarrow H(X \otimes Y) \rightarrow \operatorname{Tor}_{1}(H(X), H(Y)) \rightarrow 0
$$

where all the homomorphisms are natural, the first having degree 0 and the second having degree -1 . If $X$ and $Y$ are both free, then the sequence splits.

Theorem 1.3. Same as Theorem 1.2, but replace "degree -1" by "degree 1" throughout.

Proof. These are standard Künneth theorems for chain complexes and a proof may be found in [5]. A particularly simple proof is given by Heller in [7].

\section{Theorem 1.4.}

(a) Let $C_{i}$ and $D_{i}$ be right and left $R_{i}$ modules, respectively, where $R_{i}$ is a ring with unit and $i=1,2$. Let $\Lambda$ be a subring of the center of both $R_{1}$ and $R_{2}$. Then there exists a unique natural isomorphism

$$
T:\left[C_{1} \otimes_{R_{1}} D_{1}\right] \otimes_{\Lambda}\left[C_{2} \otimes_{R_{2}} D_{2}\right] \rightarrow\left[C_{1} \otimes_{\Lambda} C_{2}\right] \otimes_{R}\left[D_{1} \otimes_{\Lambda} D_{2}\right],
$$

where $R=R_{1} \otimes_{\Lambda} R_{2}$.

(b) If $C_{i}$ and $D_{i}$ are chain complexes and either $C_{1}$ and $C_{2}$ or $D_{1}$ and $D_{2}$ have the trivial grading, then $T$ is an isomorphism of chain complexes.

Proof. We prove (a) by showing that both groups are solutions of essentially the same "universal mapping problem".

$\left(a_{1}\right)$ The left side of $(*)$ is the solution to the universal mapping problem

$$
f: C_{1} \times D_{1} \times C_{2} \times D_{2} \rightarrow A,
$$

where $A$ is an arbitrary abelian group and $f$ is a group homomorphism satisfying

$$
f\left(c_{1} r_{1}, d_{1}, c_{2} r_{2}, d_{2}\right)=f\left(c_{1}, r_{1} d_{1}, c_{2}, r_{2} d_{2}\right)
$$

for all $r_{i} \in R_{i}$, and

$$
f\left(c_{1}, d_{1} \lambda, c_{2}, d_{2}\right)=f\left(c_{1}, d_{1}, \lambda c_{2}, d_{2}\right),
$$

for all $\lambda \in \Lambda$. This may be seen as follows. If we take $c_{2}$ to be the unit in $R_{2}$, the formula (1) asserts that $f$ is really a map $\left[C_{1} \otimes_{R_{1}} D_{1}\right] \times\left[C_{2} \times D_{2}\right] \rightarrow A$. Similarly, if we take $c_{1}$ to be the unit in $R_{1}$, we get that $f$ is a map

$$
\left[C_{1} \otimes_{R_{1}} D_{1}\right] \times\left[C_{2} \otimes_{R_{2}} D_{2}\right] \rightarrow A
$$

To see that $f$ is compatible with the action of $\Lambda$, we need only note that each $\lambda \in \Lambda$ commutes with every element in both $R_{1}$ and $R_{2}$ and then use formula (2). 
$\left(a_{2}\right)$ The right side of $(*)$ is the solution to the mapping problem

$$
g: C_{1} \times C_{2} \times D_{1} \times D_{2} \rightarrow B
$$

where $B$ is an arbitrary abelian group and $g$ is a group homomorphism satisfying

$$
\begin{aligned}
& g\left(c_{1} \lambda, c_{2}, d_{1}, d_{2}\right)=g\left(c_{1}, \lambda c_{2}, d_{1}, d_{2}\right) \\
& g\left(c_{1}, c_{2}, d_{1} \lambda, d_{2}\right)=g\left(c_{1}, c_{2}, d_{1}, \lambda d_{2}\right)
\end{aligned}
$$

for all $\lambda \in \Lambda$, and

$$
g\left(c_{1} r_{1}, c_{2} r_{2}, d_{1}, d_{2}\right)=g\left(c_{1}, c_{2}, r_{1} d_{1}, r_{2} d_{2}\right) .
$$

As before, this is checked easily and follows from the fact that $\Lambda$ is a subring of the center of both $R_{1}$ and $R_{2}$. This problem, however, is trivially equivalent to the problem $g$ : $C_{1} \times D_{1} \times C_{2} \times D_{2} \rightarrow B$ where conditions (3) and (4) are altered only by transposing the variables to obtain conditions $\left(3^{\prime}\right)$ and $\left(4^{\prime}\right)$. When this is done, we find trivially that the two mapping problems are equivalent. The existence and uniqueness of $T$ follows in the usual way.

(b) This assertion follows immediately by computing $\partial T$ and $T \partial$.

Remark 1.5. The two conditions in (3) could be reduced to one as in (1) if we assumed that $\Lambda$ contained a unit.

We are now in a position to prove the Künneth Theorems. We give the details for the case of homology and indicate the proof for cohomology. Furthermore, to simplify the discussion, we confine ourselves to cell-complexes (see [12] for a definition). This means that for complexes $K_{1}$ and $K_{2}$, we may identify the chain groups $C\left(K_{1} \times K_{2}\right)$ and $C\left(K_{1}\right) \otimes C\left(K_{2}\right)$.

For arbitrary arcwise connected spaces which have a universal covering space, the theorems still hold, but we would have to use a natural chain equivalence between these chain complexes. Such an equivalence is furnished by the Eilenberg-Zilber Theorem.

Theorem 1.6. Let $K_{1}$ and $K_{2}$ be arcwise connected cell-complexes having universal coverings and $K_{3}=K_{1} \times K_{2}$. Let $G_{i}$ be right modules over $\pi_{i}=\pi_{1}\left(K_{i}\right)$, where $G_{3}=G_{1} \otimes G_{2}$ and the action of $\pi_{3}$ on $G_{3}$ is the "product action". If $G_{1}$ or $G_{2}$ is a free abelian group, then there exists a natural exact sequence

$$
\begin{aligned}
0 \rightarrow H_{*}\left(K_{1}, G_{1}\right) \otimes H_{*}\left(K_{2}, G_{2}\right) & \rightarrow H_{*}\left(K_{3}, G_{3}\right) \\
& \rightarrow \operatorname{Tor}\left[H_{*}\left(K_{1}, G_{1}\right), H_{*}\left(K_{2}, G_{2}\right)\right] \rightarrow 0,
\end{aligned}
$$

where all homology is with local coefficients and the maps are of degree 0 and -1 . If $G_{1}$ and $G_{2}$ are both free, then the sequence splits.

Proof. Let $\tilde{K}_{i}$ be the universal covering space of $K_{i}$ and note that $\tilde{K}_{1} \times \tilde{K}_{2}=\tilde{K}_{3}$. By Theorem 1.1,

$$
H_{*}\left(K_{i}, G_{i}\right) \approx H_{*}\left(G_{i} \otimes_{\pi_{i}} C\left(\tilde{K}_{i}\right)\right) .
$$

Since $C\left(\tilde{K}_{i}\right)$ is free and at least one of the two groups $G_{1}, G_{2}$ is free, we may apply 
Theorem 1.2 to obtain the exact sequence

$$
\begin{aligned}
0 \rightarrow H_{*}\left(K_{1}, G_{1}\right) \otimes H_{*}\left(K_{2}, G_{2}\right) & \rightarrow H_{*}\left[\left(G_{1} \otimes_{\pi_{1}} C\left(\tilde{K}_{1}\right)\right) \otimes\left(G_{2} \otimes_{\pi_{2}} C\left(\tilde{K}_{2}\right)\right)\right] \\
& \rightarrow \operatorname{Tor}\left[H_{*}\left(K_{1}, G_{1}\right), H_{*}\left(K_{2}, G_{2}\right)\right] \rightarrow 0 .
\end{aligned}
$$

Recall that $Z\left(\pi_{1} \times \pi_{2}\right) \approx Z\left(\pi_{1}\right) \otimes Z\left(\pi_{2}\right)$. Thus, if we take $R_{i}=Z\left(\pi_{i}\right)$ and $\Lambda=Z$ in Theorem 1.4, we obtain the following natural isomorphisms

$$
\begin{aligned}
H_{*}\left(G_{3} \otimes_{R_{3}} C\left(\tilde{K}_{1} \times \tilde{K}_{2}\right)\right) & \approx H_{*}\left[\left(G_{1} \otimes G_{2}\right) \underset{R_{1} \otimes R_{2}}{\otimes}\left(C\left(\tilde{K}_{1}\right) \otimes C\left(\tilde{K}_{2}\right)\right)\right] \\
& \approx H_{*}\left[\left(G_{1} \otimes_{\pi_{1}} C\left(\tilde{K}_{1}\right)\right) \otimes\left(G_{2} \otimes_{\pi_{2}} C\left(\tilde{K}_{2}\right)\right)\right] .
\end{aligned}
$$

The desired sequence is obtained by substituting in the previous sequence.

The last assertion of the theorem follows from the corresponding assertion of Theorem 1.2, since $G_{i}$ free for $i=1,2$ implies $G_{i} \otimes_{\pi_{1}} C\left(\tilde{K}_{i}\right)$ is also free.

Theorem 1.7. Let $K_{i}, \pi_{i}, G_{i}$ be as before except that $G_{i}$ is a left $\pi_{i}$ module and $C_{q}\left(K_{i}\right)$ is finitely generated for each $q$. If either $G_{1}$ or $G_{2}$ is free, then there exists a natural exact sequence

$$
\begin{aligned}
0 \rightarrow H^{*}\left(K_{1}, G_{1}\right) \otimes H^{*}\left(K_{2}, G_{2}\right) & \rightarrow H^{*}\left(K_{3}, G_{3}\right) \\
& \rightarrow \operatorname{Tor}\left[H^{*}\left(K_{1}, G_{1}\right), H^{*}\left(K_{2}, G_{2}\right)\right] \rightarrow 0,
\end{aligned}
$$

where the first map has degree 0 and the second has degree 1 . If $G_{1}$ and $G_{2}$ are both free, the sequence splits.

Proof. The proof is similar to the proof of Theorem 1.6, this time using the second formula of Theorem 1.1 and then Theorem 1.3. We need an analogue of Theorem 1.4, which is furnished by the following lemma.

Lemma 1.8. Let $C_{i}$ and $D_{i}$ be left $\pi_{i}$ modules with $C_{3}=C_{1} \otimes C_{2}, D_{3}=D_{1} \otimes D_{2}$ and $\pi_{3}=\pi_{1} \times \pi_{2}$. Suppose $C_{i}$ is a free chain complex and finitely generated in each dimension. Then there is a natural chain isomorphism

$$
\operatorname{Hom}_{\pi_{1}}\left(C_{1}, D_{1}\right) \otimes \operatorname{Hom}_{\pi_{2}}\left(C_{2}, D_{2}\right) \rightarrow \operatorname{Hom}_{\pi_{3}}\left(C_{1} \otimes C_{2}, D_{1} \otimes D_{2}\right) .
$$

Proof. One merely verifies that the map given by

$$
\left(f_{1} \otimes f_{2}\right)\left(c_{1} \otimes c_{2}\right)=\left(f_{1} c_{1}\right) \otimes\left(f_{2} c_{2}\right)
$$

has the desired properties.

Remark 1.9. The requirement in Theorem 1.7 that one of the groups $G_{1}, G_{2}$ is free is needed to insure that one of the groups $\operatorname{Hom}_{\pi_{i}}\left(C\left(\tilde{K}_{i}\right), G_{i}\right)$ is free, so Theorem 1.3 applies.

\section{Twisted integral cohomology}

In this section, we consider local systems on a space with the group $Z$ of integers as the fiber. Then $\Gamma(Z) \approx Z_{2}$. Given a space $K$ which is arcwise connected, a local system with fiber $Z$ is given by an element of $\operatorname{Hom}\left(\pi_{1}(K), Z_{2}\right.$ ) as before (see Section 1). Since the Hurewicz map induces the isomorphism $\operatorname{Hom}\left(\pi_{1}(K), Z_{2}\right) \approx$ 
$\operatorname{Hom}\left(H_{1}(K), Z_{2}\right)$ and by the Universal Coefficient Theorem $\operatorname{Hom}\left(H_{1}(K), Z_{2}\right) \approx$ $H^{1}\left(K, Z_{2}\right)$, we may identify such a local system with an element $v \in H^{1}\left(K, Z_{2}\right)$. We call such a system of coefficients "twisted" and the corresponding cohomology groups $H^{q}\left(K, Z_{v}\right)$ are twisted integral cohomology groups. Note that if $v=0$, we get ordinary cohomology $[\mathbf{1 2}]$.

\subsection{The Bockstein Coboundary}

Given a twisted local system $Z_{v}$ on a space $K$, by the Bockstein coboundary $\beta_{v}$ we mean the coboundary induced by the exact sequence $0 \rightarrow Z_{v} \stackrel{2}{\rightarrow} Z_{v} \stackrel{\rho}{\rightarrow} Z_{2} \rightarrow 0$, where $Z_{2}$ is the group of integers mod 2 and the maps are multiplication by 2 and reduction. Define $\bar{\beta}_{v}=\rho \circ \beta_{v}$.

Definition 2.1. For any space $K$, define

$$
\bar{\beta}: H^{1}\left(K, Z_{2}\right) \times H^{q}\left(K, Z_{2}\right) \rightarrow H^{q+1}\left(K, Z_{2}\right)
$$

by

$$
\bar{\beta}(v, x)=\bar{\beta}_{v}(x) .
$$

Lemma 2.2. $\bar{\beta}$ is a cohomology operation in two variables.

Proof. We must show that for any continuous map $g: K^{\prime} \rightarrow K$ and elements $v \in H^{1}\left(K, Z_{2}\right), x \in H^{q}\left(K, Z_{2}\right)$, we have $g^{*} \bar{\beta}(v, x)=\bar{\beta}\left(g^{*} v, g^{*} x\right)$. Let $v \in H^{1}\left(K, Z_{2}\right)$ be fixed and $Z_{v}, Z_{g^{*}}$ be the indicated local coefficient systems over $K$ and $K^{\prime}$, where $g^{*} v \in H^{1}\left(K^{\prime}, Z_{2}\right)$. Then, it is clear that $Z_{g^{*} v}$ is the local system over $K^{\prime}$ induced by $g: K^{\prime} \rightarrow K$ in the usual sense and it follows from the usual properties of the Bockstein homomorphism [12] that $g^{*} \beta_{v}(x)=\beta_{g^{*} v}\left(g^{*} x\right)$. Since $g^{*}$ also commutes with reduction, we get $g^{*} \bar{\beta}(v, x)=g^{*} \bar{\beta}_{v}(x)=\bar{\beta}_{g^{*} v}\left(g^{*}(x)\right)=\bar{\beta}\left(g^{*} v, g^{*} x\right)$ which completes the proof of the Lemma.

Theorem 2.3. $\bar{\beta}(v, x)=v x+S q^{1} x$.

Proof. By looking at the universal example $K\left(Z_{2}, 1\right) \times K\left(Z_{2}, q\right)$ for such operations (see for example $[\mathbf{1 3}]$ ), we find immediately that

$$
\bar{\beta}(v, x)=a_{q} v^{q+1}+b_{q} v x+c_{q} S q^{1} x,
$$

where $q$ is the degree of $x$ and $a_{q}, b_{q}, c_{q} \in Z_{2}$ depend only on $q$.

We note that for any space, if $v=0$, then $Z_{v}$ is ordinary integral cohomology and $\bar{\beta}_{0}=S q^{1}$. Thus we have

$$
S q^{1}(x)=\bar{\beta}(0, x)=c_{q} S q^{1} x \Rightarrow c_{q}=1 .
$$

On the other hand, we have

$$
0=\bar{\beta}(v, 0)=a_{q} v^{q+1} \Rightarrow a_{q}=0,
$$

and

$$
\bar{\beta}(v, x)=b_{q} v x+S q^{1} x .
$$

In particular,

$$
\bar{\beta}\left(v, v^{q}\right)=b_{q} v^{q+1}+S q^{1} v^{q}=b_{q} v^{q+1}+q v^{q+1} \bmod 2 .
$$


But it is a trivial computation that for $v=w_{1} \in H^{*}\left(B O(1), Z_{2}\right)=H^{*}\left(Z_{2}, 1, Z_{2}\right)$, we have $\bar{\beta}\left(w_{1}, w_{1}^{q}\right)=(q+1) w_{1}^{q+1} \bmod 2$. It follows that $b_{q}=1$ and the theorem is proved.

\subsection{The Gysin sequence of a sphere bundle}

Let $\left(E, \pi, B, S^{k-1}\right)$ be a fiber bundle where $k \geqslant 1$. Then Thom has proved [14] that there exist Gysin sequences

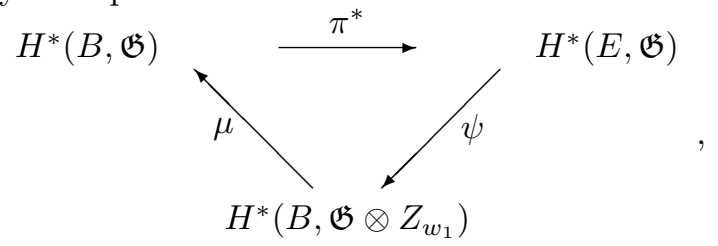

where $\mathfrak{G}$ is any local system with fiber $G$ over $B$ and we also write $\mathfrak{G}$ for the system induced on $E$ by $\pi: E \rightarrow B$. The local system $Z_{w_{1}}$ is the one determined by the first Stiefel-Whitney class of the bundle. Furthermore, if $G=Z$, then $\mu$ is given by $\mu(x)=W_{k} x$ where $W_{k} \in H^{k}\left(B, Z_{w_{1}}\right)$ is the "Euler class" of the bundle. Note that $W_{k}$ is an ordinary cohomology class if and only if $w_{1}=0$.

In what follows, we restrict ourselves to the case $G=Z$. In this case, we actually obtain two Gysin sequences

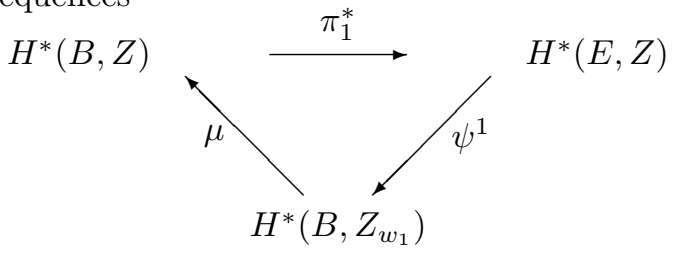

and

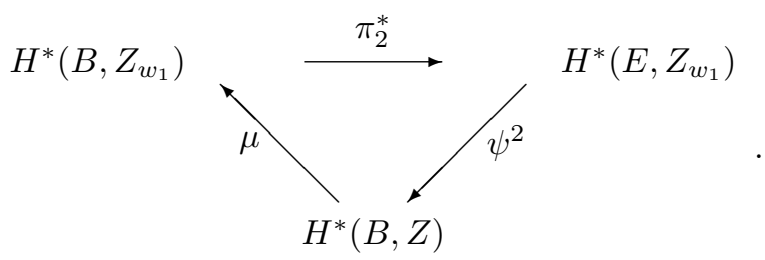

Theorem 2.4. $\psi^{1}$ and $\psi^{2}$ have the following multiplicative properties:

$$
\begin{aligned}
& \psi^{i}\left[\left(\pi_{i}^{*} x\right) y\right]=(-1)^{p} x\left(\psi^{i} y\right) \\
& \psi^{i}\left[y\left(\pi_{i}^{*} x\right)\right]=(-1)^{k p} x\left(\psi^{i} y\right),
\end{aligned}
$$

where $p$ is the degree of $x$ and $k \geqslant 1$ if $i=1$ and $k \geqslant 2$ if $i=2$.

The proof is exactly the same as the proof of Lemma 1 in $[\mathbf{8}]$ since all the properties of the maps that Massey used are still valid with cohomology in a local system (see [13]). The one exception is the case $k=1$ and $i=2$. Here, the proof in [8] breaks down because the $S^{0}$-bundle $E \rightarrow B$ is precisely the one which "kills off" the element $w_{1} \in H^{1}\left(B, Z_{2}\right)$. Thus, the local system induced by $Z_{w_{1}}$ on $E$ is not twisted. For this case, we have the following result.

Theorem 2.5. $\psi^{2} \pi_{1}^{*}(x)=2 x$ if $w_{1} \neq 0$. 
Proof. Define $\Theta\left(w_{1}, x\right)=\psi^{2} \pi_{1}^{*}(x)$. We shall show that $\Theta$ is a cohomology operation in two variables.

Note that for any $v \in H^{1}\left(B, Z_{2}\right)$, we can define the 0 -sphere bundle $\pi_{v}: E_{v} \rightarrow B$ which "kills off" the element $v \in H^{1}\left(B, Z_{2}\right)$. Then, the two Gysin sequences are defined as before and so is $\Theta(v, x)$. The verification of the fact that $\Theta$ is a cohomology operation proceeds as in the case of the Bockstein coboundary using the fact that the maps in the Gysin sequence commute with bundle maps.

(a) $\Theta(0, x)=0$ :

This is a triviality since in this case, the two Gysin sequences are the same and the result follows by exactness.

(b) Computation of $\Theta(v, x)$ for $v \neq 0$ :

It is sufficient to compute for the universal example which in this case is $U_{n}=K\left(Z_{2}, 1\right) \times K(Z, n)$ and $\Theta$ is a map

$$
\Theta: H^{1}\left(U_{n}, Z_{2}\right) \times H^{q}\left(U_{n}, Z\right) \rightarrow H^{q}\left(U_{n}, Z\right) .
$$

If we compute by means of the Künneth formula, we find that for $n \geqslant 2$

$$
\begin{gathered}
H^{1}\left(U_{n}, Z_{2}\right) \approx H^{1}\left(Z_{2}, 1, Z_{2}\right) \\
H^{n}\left(U_{n}, Z\right) \approx H^{n}\left(Z_{2}, 1, Z\right) \oplus H^{n}(Z, n, Z) .
\end{gathered}
$$

If $\nu \in H^{1}\left(K\left(Z_{2}, 1\right), Z_{v}\right)$ is the non-zero element then $H^{*}\left(Z_{2}, 1, Z\right)=Z\left[\nu^{2}\right]$ is the polynomial ring in the one generator $\nu^{2}$. Furthermore, $\nu=v \bmod 2$. We also have that $H^{n}(Z, n, Z)=Z$. Let $u_{n} \in H^{n}(Z, n, Z)$ be a generator. Then, we get immediately that $\Theta(v, x)=a_{n} x$ for $n$ odd and some $a_{n} \in Z$ and $\Theta(v, x)=b_{n} \nu^{n}+a_{n} x$ for $n$ even and some $b_{n} \in Z_{2}, a_{n} \in Z$. Since $\Theta(v, 0)=0$, we have

$$
\Theta(v, x)=a_{n} x,
$$

where $n$ is the degree of $x$.

Looking at the Gysin sequences corresponding to $v \in H^{1}\left(U_{n}, Z\right)$, we find that the two-fold covering is the universal covering $\tilde{U}_{n}=\tilde{K}\left(Z_{2}, 1\right) \times K(Z, n)$. Thus, $H^{q}\left(U_{n}, Z\right) \approx H^{q}(Z, n)$ for all $q$. In particular, $H^{n}\left(\tilde{U}_{n}, Z\right) \approx H^{n}(Z, n)$. Furthermore, since $\nu \in H^{1}\left(U_{n}, Z\right)$ is of order 2 , we have $\mu\left(2 u_{n}\right)=0$ and hence $u_{n}$ is in the image of $\psi^{2}$ by exactness of the sequence (G2). However, since $\psi^{2}$ is an additive map, it follows that $\psi^{2}\left(u_{n}\right)=2 u_{n}$. If we now turn to the sequence (G1), we easily compute $H^{n-1}\left(U_{n}, Z_{v}\right)$ by the Künneth formula and find that $\pi_{1}^{*}\left(u_{n}\right)=u_{n}$. It follows that $\psi^{2} \pi_{1}^{*} u_{n}=2 u_{n}$ and therefore $\Theta(v, x)=2 x$.

For the case $n=1$, we can compute separately. Here, we note that $K\left(Z_{2}, 1\right)=$ $P_{\infty}(R)$ is infinite-dimensional real projective space and the universal example is $P_{\infty}(R) \times P_{\infty}(R)$. But in this case, all the groups in the Gysin sequence are known and we can compute $\psi^{2}$ directly.

\section{Cohomology of $B O(n)$}

In this section, we apply the preceding results to determine completely the structure of the cohomology ring $H^{*}(B O(n), Z)$ as well as the complete structure of 
$H^{*}(B O(n), \mathfrak{Z})$, where $\mathfrak{Z}$ is the local system $Z_{w_{1}}$. We describe these groups in terms of the cohomology ring $H^{*}(B S O(n), Z)$ and the two Gysin sequences associated with the covering $B S O(n) \rightarrow B O(n)$. To complete the picture, we give a formula in closed form for the ring $H^{*}(B S O(n), Z)$.

\subsection{Torsion in $H^{*}(B O(n), \mathfrak{Z})$}

It has been shown by Borel and Hirzebruch [3] that all of the torsion in the groups $H^{*}(B O(n), Z)$ and $H^{*}(B S O(n), Z)$ is of order 2. Using these results, we prove

Theorem 3.1. All the torsion in $H^{*}(B O(n), \mathfrak{Z})$ is of order 2 .

Proof. Consider the following diagram

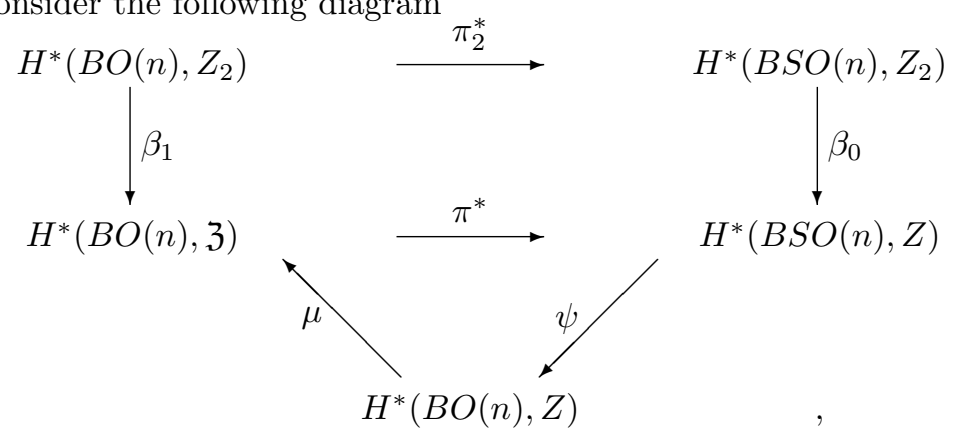

where $\pi: B S O(n) \rightarrow B O(n)$ is the two-fold covering, $\beta_{0}$ and $\beta_{1}=\beta_{w_{1}}$ are Bockstein homomorphisms, and the bottom triangle is the Gysin sequence. The top square commutes by virtue of Lemma 2.2 and it is known that $\pi_{2}^{*}$ is onto. In fact, if $w_{j}$ are the mod 2 Stiefel-Whitney classes then

$$
\begin{aligned}
H^{*}\left(B O(n), Z_{2}\right) & \approx Z_{2}\left[w_{1}, \ldots, w_{n}\right] \\
H^{*}\left(B S O(n), Z_{2}\right) & \approx Z_{2}\left[w_{2}, \ldots, w_{n}\right]
\end{aligned}
$$

and $\pi_{2}^{*}$ is given by $\pi_{2}^{*}\left(w_{j}\right)=w_{j}$ for $j \neq 1$, and $\pi_{2}^{*}\left(w_{1}\right)=0$. (See, for example, [9]). Suppose $x \in H^{q}(B O(n), \mathfrak{Z})$ and $k x=0$ for some non-zero integer $k$. Then, $0=\pi^{*}(k x)=k \pi^{*} x$ implies

$$
\pi^{*} x=0 \quad \text { or } \quad 2 \pi^{*} x=0,
$$

since $H^{*}(B S O(n), Z)$ has torsion only of order 2 .

Suppose $\pi^{*} x=0$. Then, by exactness of the triangle, $x=\mu(y)=\mathfrak{w}_{1} y$, where $\mathfrak{w}_{1} \in H^{1}(B O(n), \mathfrak{Z})$ is the non-zero element. But then $2 x=2 \mathfrak{w}_{1} y=0$.

If $2 \pi^{*} x=0$, then $\exists u \in H^{*}\left(B S O(n), Z_{2}\right)$ with $\pi^{*} x=\beta_{0} u$ by exactness of the Bockstein sequence. But $\pi_{2}^{*}$ is onto, so there exists $v$ such that $\pi_{2}^{*} v=u$. Then,

$$
\pi^{*} \beta_{1} v=\beta_{0} \pi_{2}^{*} v=\beta_{0} u=\pi^{*} x
$$

and $\pi^{*}\left(\beta_{1} v-x\right)=0$. As before, this implies $0=2\left(\beta_{1} v-x\right)=2 \beta_{1} v-2 x$. But $2 \beta_{1} v$ $=0$ and so $2 x=0$.

Theorem 3.2. For $n$ odd, $H^{*}(B O(n), \mathfrak{Z})$ is a direct sum of copies of $Z_{2}$. 
Proof. For $n=1$, this is well known since $B O(1)=P_{\infty}(R)$ is infinite-dimensional real projective space. For $n \geqslant 3$, we have $O(n)=S O(n) \times O(1)$ and hence $B O(n)=$ $B S O(n) \times B O(1)$. Applying the Künneth Theorem of Section 1, we get a split exact sequence. Next we note that $H^{p}(B S O(k), Z)$ is a direct sum of copies of $Z$ and $Z_{2}$. On the other hand,

$$
H^{q}(B O(1), \mathfrak{Z})=\left\{\begin{array}{ll}
0 & q \text { even } \\
Z_{2} & q \text { odd }
\end{array} .\right.
$$

This may be seen as follows. As noted earlier, $B O(1)=K\left(Z_{2}, 1\right)=P_{\infty}(R)$. That $H^{0}(B O(1), \mathfrak{Z})=0$ follows from [12, Section 31.20]. For the higher groups, we may approximate $P_{\infty}(R)$ by $P_{2 q}(R), 2 q$-dimensional projective space over the reals, since this is the $2 q$-skeleton. But for a compact non-orientable manifold, we may compute the twisted cohomology from the ordinary homology by the Poincaré duality theorem [4, exposé 20].

\subsection{The twisted Euler class}

For orientable bundles with fiber $S^{k-1}$, the Whitney sum theorem asserts that $X(\xi \oplus \eta)=X(\xi) X(\eta)$ where $\xi \oplus \eta$ is the Whitney sum of the two bundles and $X(\zeta)$ is the Euler class of $\zeta$ (see $[\mathbf{2}]$ or [9]). In this section, we prove the corresponding theorem for non-orientable bundles.

Let $p: B O(n-1) \rightarrow B O(n)$ be the $(n-1)$-sphere bundle induced by the inclusion $O(n-1) \rightarrow O(n)([\mathbf{2}])$. According to Thom [14], we have the Gysin sequences (G1) and (G2) as in Section 2.2 and the map $\mu$ is multiplication by a fixed element $\mathfrak{w}_{n} \in H^{n}(B O(n), \mathfrak{Z})$. Furthermore, it is also shown in [14] that $\mathfrak{w}_{n}$ is equal to the first obstruction to a cross-section of the bundle (see [12]) with a change of sign. The class $\mathfrak{w}_{n}$ (or its negative) is usually called the universal twisted Euler class. As usual, the Euler class of a sphere bundle is defined by means of the classifying map of the bundle $[\mathbf{9}]$.

Theorem 3.3. For any two sphere bundles $\xi, \eta$ (not necessarily orientable) the Whitney sum theorem is valid. That is $\chi(\xi) \cdot \chi(\eta)=\chi(\xi \oplus \eta)$, where $\chi(\zeta)$ is the Euler class of the bundle $\zeta$.

Proof. It is sufficient to check this for the universal classes. Let

$$
\pi: B O(n) \times B O(m) \rightarrow B O(n+m)
$$

be the fibering induced by the inclusion $O(n) \times O(m) \rightarrow O(n+m)$ (as in [12]). We then obtain the following diagram:

$$
\begin{aligned}
& H^{n+m}(B O(n+m), \Re) \stackrel{\rho}{\leftarrow} H^{n+m}(B O(n+m), \mathfrak{Z}) \stackrel{\rho_{2}}{\rightarrow} H^{n+m}\left(B O(n+m), Z_{2}\right) \\
& \downarrow \pi^{*} \quad \rho \quad \downarrow \pi^{*} \quad \rho_{2} \quad \downarrow \pi^{*} \\
& H^{n+m}(B O(n) \times B O(m), \mathfrak{R}) \stackrel{\rho}{\leftarrow} H^{n+m}(B O(n) \times B O(m), \mathfrak{Z}) \stackrel{\rho_{2}}{\rightarrow} H^{n+m}\left(B O(n) \times B O(m), Z_{2}\right),
\end{aligned}
$$

where $\mathfrak{R}$ is the local system of twisted rationals, and $\rho$ and $\rho_{2}$ are induced by the coefficient homomorphisms of inclusion and reduction mod 2.

$$
\rho_{2} \pi^{*} \mathfrak{w}_{\mathrm{n}+\mathrm{m}}=\rho_{2}\left(\mathfrak{w}_{\mathrm{n}} \otimes \mathfrak{w}_{\mathrm{m}}\right) .
$$

The assertion is simply that the Whitney sum theorem holds for the mod 2 Euler 
class (top Stiefel-Whitney class) since $\rho_{2} \mathfrak{w}_{n+m}=w_{n+m}$. This is proved, for example, in $[\mathbf{9}]$.

$$
\rho \pi^{*} \mathfrak{w}_{\mathrm{n}+\mathrm{m}}=\rho\left(\mathfrak{w}_{\mathrm{n}} \otimes \mathfrak{w}_{\mathrm{m}}\right) .
$$

If either $n$ or $m$ is odd, this is a triviality since one computes by the Künneth formula and Theorem 3.1 that $H^{n+m}(B O(n) \times B O(m), \mathfrak{R})=0$.

Suppose both $n$ and $m$ are even and let $j_{q}: B S O(q) \rightarrow B O(q)$ be the two-fold covering. Then $j_{q}^{*}\left(\mathfrak{w}_{q}\right)=W_{q} \in H^{q}(B S O(q), Z)$ by naturality of characteristic classes. But the Whitney sum theorem is known for orientable bundles [9]. Thus, it is sufficient to prove that

$$
j^{*}: H^{q}(B O(q), \mathfrak{R}) \rightarrow H^{q}(B S O(q), \mathbb{Q})
$$

is a monomorphism for $q$ even, where $\mathbb{Q}$ denotes the field of rational numbers. By exactness of the Gysin sequence of the 2-fold covering, the kernel would have to be the same as the image of

$$
H^{q-1}(B O(q), \mathbb{Q}) \stackrel{\mu}{\rightarrow} H^{q}(B O(q), \mathfrak{R}) .
$$

But for $q$ even $H^{q-1}(B O(q), \mathbb{Q})=0$ (see $\left.[\mathbf{3}]\right)$. This completes the proof of $(\mathrm{b})$.

$$
\pi^{*} \mathfrak{w}_{\mathrm{n}+\mathrm{m}}=\mathfrak{w}_{\mathrm{n}} \otimes \mathfrak{w}_{\mathrm{m}} .
$$

Since $B O(n)$ has only 2-torsion, the same holds true for $B O(n) \times B O(m)$ as can be checked by the Künneth formula. But then an element is completely determined by its image in the rationals and reduction mod 2 .

\subsection{The Gysin sequences of $B S O(n) \rightarrow B O(n)$}

In this section we determine completely the maps in the Gysin sequences of $\pi: B S O(n) \rightarrow B O(n)$ and thus determine completely the structure of the cohomology of $B O(n)$ in terms of $B S O(n)$.

We will write $p_{j}$ for the integral Pontryagin classes of $B O(n)$ and $B S O(n)$, and $\mathfrak{w}_{n}$ and $W_{n}$ for the universal twisted and ordinary Euler classes respectively.

Theorem 3.4. For $B O(n)$ and $B S O(n)$, the maps $\psi^{1}, \psi^{2}$ of the Gysin sequences (G1) and (G2) in Section 2.2 are given by:

1. $\psi^{i}(x)=0$ if $2 x=0$,

2. $\psi^{1}\left(m\left(p_{1}, \ldots, p_{n}\right)\right)=0, \psi^{1}\left(W_{n} m\left(p_{1}, \ldots, p_{n}\right)\right)=2 \mathfrak{w}_{n} m\left(p_{1}, \ldots, p_{n}\right)$,

3. $\psi^{2}\left(m\left(p_{1}, \ldots, p_{n}\right)\right)=2 m\left(p_{1}, \ldots, p_{n}\right), \psi^{2}\left(W_{n} m\left(p_{1}, \ldots, p_{n}\right)\right)=0$, where $p_{n}=\mathfrak{w}_{n}^{2}=W_{n}^{2}$ and $m$ is a monomial.

Proof. For the first assertion, it is enough to look at the mod 2 Gysin sequence and in that case $\psi=0$ because $\pi^{*}$ is onto. The second and third assertions follow, respectively, from Theorem 2.4 and Theorem 2.5 of Section 2 once we note that $\pi^{*}\left(\mathfrak{w}_{n}\right)=W_{n}$ and $\pi^{*}\left(p_{j}\right)=p_{j}$.

\section{4. $\quad$ The ring $H^{*}(B S O(n), Z)$}

We now develop a closed formula for the cohomology ring $H^{*}(B S O(n), Z)$. We shall carry through the computation only for $n$ odd. The case $n=2 k$ is then easily 
handled since the cohomology ring of $B S O(2 k)$ can be obtained from $B S O(2 k-1)$ by adjoining the Euler class $W_{2 k}$.

Let $R=R_{2 n+1}$ be the graded commutative polynomial ring over $Z$ in the generators $W_{j}, 2 \leqslant j \leqslant 2 n+1$, with the relations $2 W_{2 j+1}=0$. For any strictly increasing sequence of integers $I=\left\{j_{1}, \ldots, j_{k}\right\}$ with $1 \leqslant j_{1}<j_{k} \leqslant n$ and $k \geqslant 2$ define

$$
W_{I}=W_{2 j_{1}} W_{2 j_{2}} \cdots W_{2 j_{k}}
$$

and

$$
V_{I}=W_{I} \sum_{j \in I} \frac{W_{2 j+1}}{W_{2 j}}
$$

Theorem 3.5. $H^{*}(B S O(2 n+1), Z)$ is isomorphic to the subring $G=G_{2 n+1}$ of $R$ generated by the elements $W_{2 j+1}, W_{2 j}^{2}$ for $1 \leqslant j \leqslant n$ and $V_{I}$ for all strictly increasing sequences $I=\left\{j_{1}, \ldots, j_{k}\right\}$ as above.

Proof. Define maps

$$
\varphi_{0}: G \rightarrow H^{*}(B S O(2 n+1), \mathbb{Q}) \quad \text { and } \quad \varphi_{2}: G \rightarrow H^{*}\left(B S O(2 n+1), Z_{2}\right),
$$

where $\mathbb{Q}$ is the field of rationals, as follows. $\varphi_{0}\left(W_{2 j+1}\right)=0, \varphi_{0}\left(W_{2 j}^{2}\right)=p_{j}=j^{t h}$ Pontryagin class. Extend $\varphi_{0}$ to $G$ so that it is a ring homomorphism. We can define $\varphi_{2}: R \rightarrow H^{*}\left(B S O(2 n+1), Z_{2}\right)$ by $\varphi_{2}\left(W_{j}\right)=w_{j}=j^{t h}$ Stiefel-Whitney class and then restrict $\varphi_{2}$ to $G$. We then have the following diagram:

$$
\begin{array}{cccc}
G_{2 n+1} & \varphi_{0} & & H^{*}(B S O(2 n+1), \mathbb{Q}) \\
\varphi_{2} \downarrow & & \uparrow \rho \\
H^{*}\left(B S O(2 n+1), Z_{2}\right) & \stackrel{\rho_{2}}{ } & & H^{*}(B S O(2 n+1), Z) .
\end{array}
$$

It is clear that image $\varphi_{0}=$ image $\rho$. The theorem will be proved if we can show that image $\varphi_{2}=$ image $\rho_{2}$.

Let $F \oplus T=H^{*}(B S O(2 n+1), Z)$ be a decomposition into a free and torsion subring. That is, $T=$ Tors $H^{*}(B S O(2 n+1), Z)$ and $F$ is the subring generated by the Pontryagin classes. Then it is clear that $\rho_{2}(F \oplus T)=\rho_{2}(F) \oplus \rho_{2}(T)$. According to [3] we have that $\rho_{2}(T)=$ image $S q^{1}$ and by [1] we also have that $S q^{1}\left(w_{j}\right)=$ $j w_{j+1} \bmod 2$. By straightforward computation, since $S q^{1}$ is a derivation, we have $S q^{1}\left(w_{I}\right)=V_{I}$. Now suppose that $m\left(w_{2}, \ldots, w_{n}\right)$ is any monomial in Stiefel-Whitney classes. Then, we can write $m$ as a product of monomials $m=m_{1} \cdot m_{2} \cdot w_{2 j}$ or $m=$ $m_{1} \cdot m_{2} \cdot w_{I}$ where $m_{1}=m_{1}\left(w_{3}, w_{5}, \ldots, w_{2 n+1}\right)$ and $m_{2}=m_{2}\left(w_{2}^{2}, w_{4}^{2}, \ldots, w_{2 n}^{2}\right)$. Then $S q^{1} m m_{2}=0$ and $S q^{1} m=m_{1} m_{2} w_{2 j+1}$ or $S q^{1} m=m_{1} m_{2} V_{I}$. Since $\rho_{2}\left(p_{j}\right)=$ $w_{2 j}^{2}$ it follows that $\rho_{2}(F \otimes T)=\rho_{2}(F) \otimes \rho_{2}(T)=\varphi_{2}(G)$.

\section{References}

[1] A. Borel, La cohomologie mod 2 de certains espaces homogènes, Comm. Math. Helv., 27, 165-197, 1953.

[2] A. Borel, Selected Topics in the Homology Theory of Fibre Bundles, Dept. of Math., Univ. of Chicago, 1954, (mimeographed notes). 
[3] A. Borel and F. Hirzebruch, Characteristic Classes and Homogeneous Spaces II, Amer. J. Math., 81, 315-382, 1959.

[4] H. Cartan, Homologie des Groupes, Suite Spectrale, Faisceaux, Ecole Normale Supérieure, Paris, 1950, (mimeographed seminar notes).

[5] H. Cartan, and S. Eilenberg, Homological Algebra, Princeton University Press, Princeton, 1956.

[6] S. Eilenberg, Homology of Spaces with Operators I, Trans. AMS, 61, 378417, 1947.

[7] A. Heller, A Simple Proof of the Künneth Theorem, Proc. AMS, 11, (1960), 676-678.

[8] W. S. Massey, On the Cohomology Ring of a Sphere Bundle, Jour. of Math. and Mechanics, 7, 265-290, 1958.

[9] J. W. Milnor and J. D. Stasheff, Characteristic classes, Annals of Mathematics Studies, No. 76, Princeton University Press, Princeton, 1974.

[10] J. P. Serre, Cohomologie mod 2 des complexes d'Eilenberg-MacLane, Comm. Math. Helv., 27, 198-232, 1953.

[11] N. E. Steenrod, Homology with Local Coefficients, Annals of Math., 44, 610 627, 1943.

[12] N. E. Steenrod, Topology of Fibre Bundles, Princeton University Press, Princeton, 1951.

[13] N. E. Steenrod, Cohomology Operations, Symposium Internacional de Topología Algebraica, Mexico City, 1958.

[14] R. Thom, Espaces fibrés en sphères et carrés de Steenrod, Annales de L'école Normale Supérieure, 3-série, 69, 109-182, 1952.

[15] H. Whitney, On the topology of differentiable manifolds, Lectures in Topology, 101-141, University of Michigan Press, 1941.

Robert Greenblatt larry@uni-math.gwdg.de

c/o Larry Smith

Mathematisches Institut

Georg-August-Universität-Göttingen

37073 Göttingen

Bunsenstraß3

Federal Republic of Germany

This article is available at http://intlpress.com/HHA/v8/n2/a5 